\title{
Parameter Calibration of CT System
}

\author{
Yurui Peng \\ North China Electric Power University, Baoding 071000, China \\ 739408170@qq.com
}

\begin{abstract}
In this paper, we use the known template to calibrate the parameters of the two-dimensional CT system. The calibration of CT system includes three aspects: the location of the center of the system in the square pallet, the distance between the detector units, and the 180 directions of the X-ray used by the system. First, the CT system is abstracted. Then, we have established the Descartes coordinate system, and the quantitative processing of the CT system is based on this coordinate system. The final solution is: The location of the center of the system is $(-9.1034,5.7931)$. The distance between the detector units is 0.2759 . The CT system is evenly rotated and the rotation angle is $1^{\circ}$. The original angle is $-60^{\circ}$. Finally, we give evaluation to our model, followed by a brief remark about our work.
\end{abstract}

Key words: CT; X-ray; square pallet; detector units; Descartes coordinate system.

\section{INTRODUCTION}

CT technology is an emerging technology that blend of the Ray optoelectronics, microelectronics and informatics subject. Because of its advanced nondestructive testing technology, it is widely used in medical, aerospace, biological and other fields. [1]

There are always many errors in the installation of CT system, which affects the imaging quality. Thus, we need to calibrate the CT system. We can calibrate the parameters of CT system with the samples of known structure, and then we will make imaging for unknown structure samples.

A common two-dimensional CT system, as shown in Fig. 1. There are 512 isometric units on a plane, and the parallel incident X-rays are perpendicular to the plane. The relative position of the transmitter and detector of the Xray is fixed, and the whole transmitting and receiving system revolves 180 times counter clockwise around a fixed center of rotation. For every X-ray direction, the detector can measure the ray energy absorbed and attenuated by twodimensional media to be fixed and get 180 sets of receiving information after gain processing.

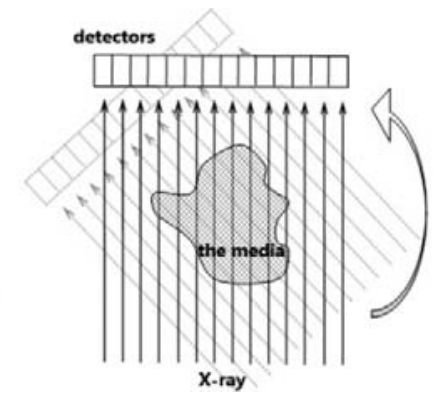

FIGURE 1. Two-dimensional CT system 


\section{PARAMETER CALIBRATION OF CT SYSTEM BASED ON TEMPLATE}

A calibration template composed of two homogeneous solid media is placed on a square tray, and the geometric information of the template is shown in Fig. 2. Based on this template, we get the received data from the template.

Taking the plane of the template as the plane of coordinates, the center of the ellipse is the origin, the ellipse axis points to the circular direction of the $\mathrm{X}$ axis, and the direction of the elliptical short axis is the $\mathrm{Y}$ axis to establish the Cartesian coordinate system, as shown in Fig. 3.

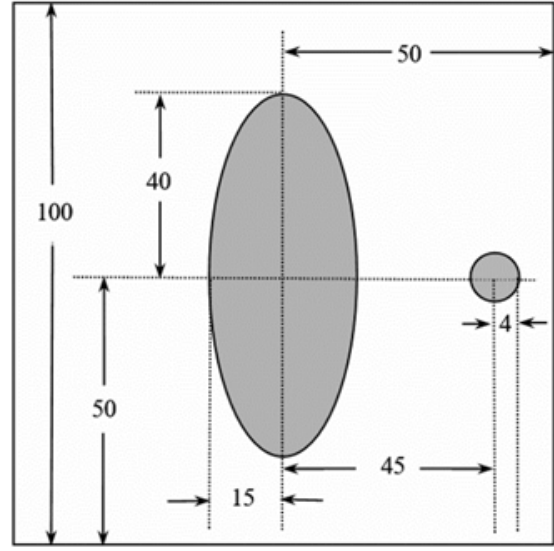

FIGURE 2. Template

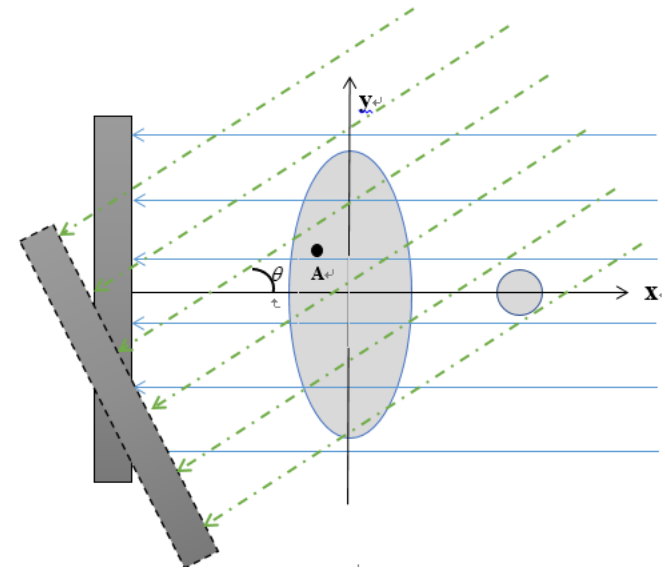

FIGURE 3. Cartesian coordinate system

Drawing the data with MATLAB to get the diagram as shown in Fig. 4. Suppose that the point in Fig. 4 is a receiving matrix $A(i, j) . A(i, j)$ represents absorptivity. According to Fig. 4, we can get the following conclusions:

- The edge of the curve is smooth without mutation, which indicates that the 180 rotation angles are uniformly changed.

- If the $\mathrm{CT}$ system can rotate more than 1 cycles, the image must change periodically. In fact, there is no periodic law of the image, and the rotation angle of the 180 rotation is less than 360 degrees.

- $\theta$ represents the angle of incidence of X-rays .It can be found that there are the widest and narrowest cases in the image. The widest case is that when $\theta=0^{\circ}$, the projection of the medium on the detector is the longest. The narrowest case is that when $\theta=90^{\circ}$, the projection of the medium on the detector is the shortest.

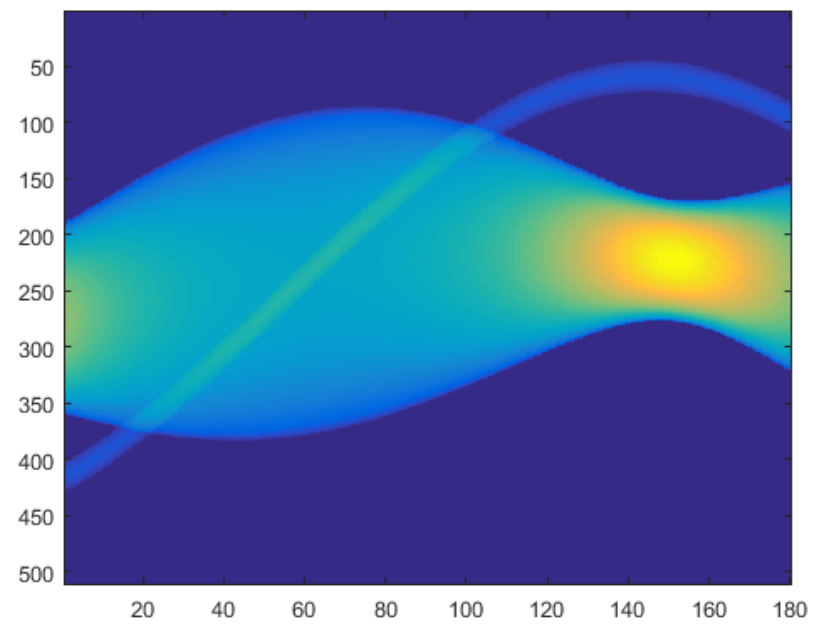

FIGURE 4. Reconstruction algorithm 
Then, we use MATLAB to calculate $\max \{A(i, j)\}$ of each rotation angle and draw the characteristic diagram as shown in Fig. 5.

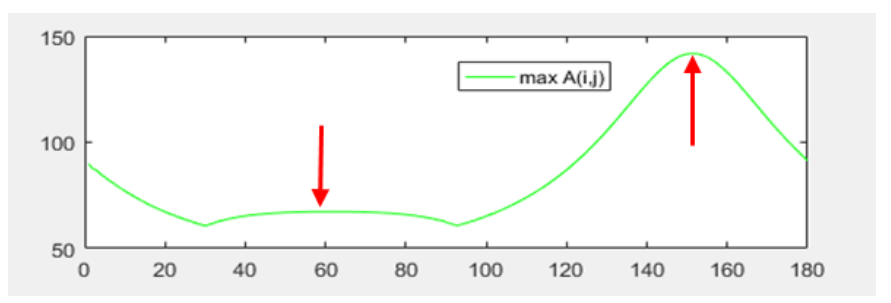

FIGURE 5. $\max A(i, j)$ characteristic diagram

According to Fig. 5, we take advantage of the two special states of $\theta=0^{\circ}$ and $\theta=90^{\circ}$. We found the following characteristics:

When $\theta=0^{\circ}, \mathrm{i}=\mathrm{u}, \mathrm{j}=\mathrm{p}$

$$
\exists \max \{\max A(i, j)\}
$$

When $\theta=90^{\circ}, \mathrm{i}=\mathrm{v}, \mathrm{j}=\mathrm{q}$

$$
\exists\{\max A(i, j)\}^{\prime}=0, \max A(i, j) \neq \max \{\max A(i, j)\}
$$

Where:

$\mathrm{i}$ represents the $\mathrm{i}$-th direction of the rotation of the CT system

$\mathrm{j}$ represents the $\mathrm{j}$-th detectors

We deal with the data and get the number of $A(i, j) \neq 0$ in each direction. Finally, we get the following conclusion:

$$
\begin{gathered}
g_{1}=\max g(i), \theta=0^{\circ} \\
g_{2}=\min g(i), \theta=90^{\circ}
\end{gathered}
$$

Where:

g represents the number of $A(i, j) \neq 0$

\section{Distance between Detector Units.}

When the incident angle of $\mathrm{X}$ ray is $90^{\circ}$, the distance between detector units can be calculated by the number of rays that are attenuated by the round shielding energy and the radius of the circle. That is:

$$
\mathrm{d}=\frac{r}{g_{1}}=\frac{8}{29}=0.2759
$$

Where:

$\mathrm{d}$ represents the distance between detector units

$\mathrm{r}$ represents the radius of the circle

\section{The 180 Directions of the CT System.}

According to Eq. 1 and Eq. 2, we get u and v. And then we calculate the angle of rotation every time.

$$
\Delta \theta=\frac{90}{u-v}=\frac{90}{151-61}=1^{\circ}
$$

Sixty-first direction is $0^{\circ}$, we recursive the original angle is $-60^{\circ}$. 


\section{Rotation Center Coordinate.}

In the field of two-dimensional image reconstruction, the central ray of parallel beam scanning passes through the center of rotation. [2] Therefore, even if the center of rotation is offset by the center of the template, the center rays must go through the center of rotation. A diagram of a rotating sketch as shown in Fig. 6.

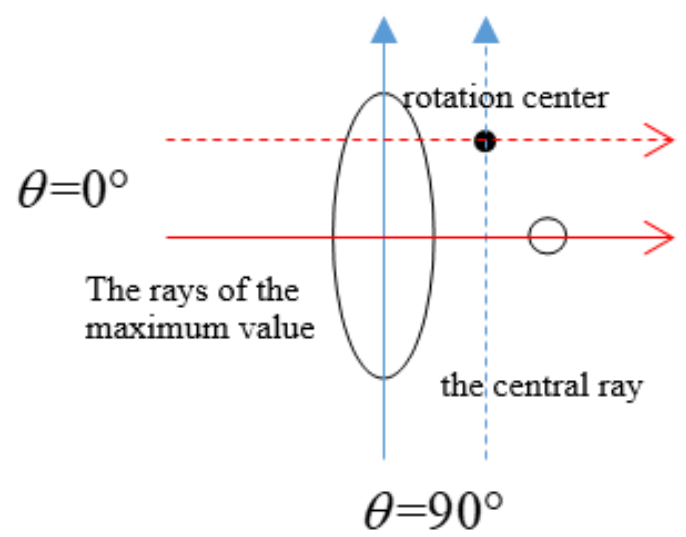

FIGURE 6. Diagram of a rotating sketch

According to Eq. 1 and Eq. 2,we get p and q, the central rays correspond to 256th detectors. Then we find abscissa of the rotation center is:

$$
x_{0}=(256-p) \times d=-9.1034
$$

And ordinate of the rotation center is:

$$
y_{0}=(256-q) \times d=5.7931
$$

Therefore, the location of the center of the system is $(-9.1034,5.7931)$.

\section{SUMMARY}

We make full use of the interconnections of the data given in the attachment, making the model more closely connected to the system. Finally, we find that the location of the center of the system is $(-9.1034,5.7931)$. The distance between the detector units is 0.2759 . The $\mathrm{CT}$ system is evenly rotated and the rotation angle is $1^{\circ}$. The original angle is $-60^{\circ}$.

\section{REFERENCES}

1. Jing Li: Journal of Henan Institute of Education (National Science Edition), Vol. 24 (2015) No.4, p.1. (In Chinese)

2. Zengyun Li and Donghui Lv: CT Theory and Applications, Vol. 24 (2015) No.4, p.533. (In Chinese).

3. Jingyu Wang, Yu Han, Lei Li, Xiaoqi Xi, Jianbang Liu, and Bin Yan: ACTA OPTICA SINCA, Vol. 37 (2017) No.8, p.1. (In Chinese).

4. Jingyu Wang, Yu Han, Lei Li, Xiaoqi Xi, Jianbang Liu, and Bin Yan: ACTA OPTICA SINCA, Vol. 37 (2017) No.8, p.2. (In Chinese).

5. Xiang Li: Research on the Application of CT Image (MS., Harbin Engineering University, China 2004), p.14. (In Chinese).

6. Xianchao Wang: Research on Local Reconstruction Algorithm of CT Image (Ph.D., Information Engineering University, China 2013), p.8. (In Chinese). 
7. Yanbin Lu: Research on X-ray CT Imaging Technology and Multi-modal Tomography (Ph.D., Peking University, China 2012), p.45. (In Chinese).

8. Zhaoba Wang: ACTA ARM AMEN TARII, Vol.22(2001) No.3, p.324. (In Chinese).

9. Jianwei Gu, Li Zhang, Zhiqiang Chen, Liang Li and Jie Tang: JTsinghua Univ (Sci\& Tech), Vol. 47 (2007) No.3, p.393. (In Chinese).

10. Jinghu Zhang, Min Guo and Yawen Wang: Computer Applications, Vol.28 (2008) No.5, p.1236. (In Chinese). 